\title{
CONTEXTUAL DEPENDENCIES AND GENDER STRATEGY
}

\author{
Peter M. Bednar \\ University of Portsmouth
}

\begin{abstract}
Analysts are often asked to help deliver systems that have a great mix of performance and features. Unfortunately, problematic organizational gender related issues have sometimes been degraded and treated as unrelated to technological issues. While information systems development in general is trying to ensure support for businesses, we might in the future expect more than just a gender-ignorant quality measure in the way the IS works to meet organizational demands. In this paper it is proposed that an interpretative and contextual analysis would support ISD in the creation of a necessary level of understanding of each specific business. The intention with an inquiry into contextual dependencies is that it helps to identify some methodological limitations which result in traps unconsciously biasing analysis ofinvestigated problem spaces. This paper introduces a contextual analysis highlighting some contextual dependencies that are typically ignored in existing works or analysis. An initial framework is proposed using gender as an example of an inquiry into some existing contextual dependencies.
\end{abstract}

Keywords: Information systems analysis, contextual dependency, contextual analysis, gender strategy

\section{INTRODUCTION}

Those problematic issues which are focused upon within this paper have to do with basic underlying theoretical assumptions in which most of our work (as systems analysts) is steeped. Both unconsciously and consciously, these issues can be assumed to have a huge impact on our everyday work. Hence they create blind spots for us and support us in being unconscious in a continuous belief of gender-neutrality in our obscured state of self-denial.

One problem is that some of the central underlying and influential issues in information systems development are dependent upon cybernetics as a base in systems 
thinking together with psychological and sociological reductionism. Another problem is that sometimes hidden political issues are related to unresolved conflicts between assumptions of observation, interaction, and intervention. The proposed strategy in this paper to breakout of this cage of self-denial is a stronger relationship (in systems analysis) to the consideration of contextual dependencies and a higher degree of reflection. One example of a problem space where inquiry into contextual dependencies become relevant is related to managerial attitude and organizational practice of promoting ideas of decentralized power in organizations.

\section{DECENTRALIZED POWER}

The presupposition that extreme economic logic has to be kept in check with a pronounced distribution of power is supported in the discussion that Galbraith, Lawler and Associates (1993) put forward about how successful new forms of organizational design and organizational structures ought to be aligned with practices that distribute information, power, knowledge, and rewards throughout the organization.

Argyris and Schön's (1978, 1996) discussion on learning organizations has put a focus on empowerment from a competence raising point of view. Argyris (1990) uses the notion of "organizational defense" to explain differences between descriptions and activities. This is a notion that might be of significant importance when efforts are made to explain why a great equal opportunity policy might not work even if management is supportive. Weick's (1995) organizational sense making can be viewed as an effort that tries to bridge the gap between strategies for

1. Analyzing - quality of interpretation and understanding

2. Designing — strategies for structuring and problem solving

Contextualism and interpretative IS research are important issues in the IS field due to the way in which several new political dimensions are put on the IS research agenda (refer to Walsham 1993; Walsham and Sahay 1999). Whatever system and ICT is to be developed is supposedly to be relevant for some particular organization and some particular organizational members that are to benefit from its implementation. This is to say that systems are possible to view as being intrinsically contextually dependent. Systems analysis in practice and theory would then have to further develop an understanding of limitations of models based on assumptions of generalizability as opposed to contextual dependency.

\section{CONTEXTUAL DEPENDENCY}

One of the major features of the combination of contextual dependency and double loop learning is the reevaluation (reflection) of a sense making process itself. Such a reflection can be seen as a metaphysical part of an enhanced version of double loop learning and is also of major importance in the sense making process as presented in the strategic systemic thinking (SST) framework proposed by Bednar (2000). This framework is proposed as a means of accessing individual and team competencies for im- 
proved systems analysis work and the intention is to provide a systemic arena for developing a learning organization inclusive of having a constructive dialogue mechanism.

The sense making processes that often might be seen as prominent in organizations are ambiguity and uncertainty. The main purpose with the SST framework is to support an adhoc creation of a systems thinking process with the aim to transfer uncertainty into structured uncertainty (this can, in some circumstances, be seen as a transformation of uncertainty into ambiguity). The impact in organizations of the two sense-making processes is somewhat different.

In the case of ambiguity, people engage in sense making because they are confused by too many interpretations, whereas in the case of uncertainty, they do so because they are ignorant of any interpretations (Weick 1995, page 91).

One of the reasons that a problematized understanding of contextual dependency is important is that some relativism is meant to be supported, but not total relativism (e.g., extreme skepticism). The intention in this paper is to avoid an attitude of anything goes without disqualifying relativistic ideas. One example where contextual dependency may be seen as related to some kind of relativistic experience is in efforts to develop and implement gender strategies.

\section{GENDER STRATEGY}

The problems with gender being a significant but understudied theme are generally widespread in British industrial relations (Wajcman 2000). Wajcman concludes that

analyses of this phenomenon have tended to be descriptive rather than explanatory. There has been increasing consciousness of the tendency to treat workers as male, but without deeper understanding of the way power inequalities between men and women shape employment relations (p. 195).

Giddens $(1984,1991)$ points out a necessity to analyze the dialectic between resistance (of socially constructed behavior) and power. There is always some (individual) possibility to influence social activities, and even if such a possibility would be viewed as minimal, it is still existent and has an impact.

Habermas (1984) suggests that individuals have ability for communicative actions and actively pursue resistance against those powers, which try to pacify the individual ability to take initiative and ability to act.

The basic perspective in this paper is that individuals as reflective subjects have an ability to break out of inhibiting social and cultural (control) systems. Argyris (1990) concludes in his discussion of organizational defenses that awareness of ability does not mean that this ability is easy to achieve in practical organizational settings.

A gender strategy in IS, related to a contextual dependency at micro and macro level analysis, could be interpreted as an effort to combine the experiences from the aforementioned contextually dependent combination of intra- and interindividual open systems thinking. 
An inquiry into contextual dependencies at a micro level can be seen as analysis of

- The specific institutional changes that might lead to a weakening of diverse intraindividual understanding of social distinctions. This as an inquiry into individual understanding of changes in relations between different types of organizational culture, which can be explored, for example, between what can be observed as male and female bias.

- How individuals might cope with experiences in their personal construction and reconstruction of biographic storytelling about their own individual gendered selves. Such a combination of construction and deconstruction assumes a reflective approach to individual sense-making and intra-individual analysis.

An inquiry into contextual dependencies at a macro level can be seen as analysis of

- The interindividual gendered tendencies toward and relations with organizational and interorganizational, technological, cultural, and social integration, not only to analyze and inquire into such processes which could then be described in terms of fragmentation and disintegration.

- How gender-related knowledge and behavior might be viewed when related to examples of specific (organizational) contexts and specific historical situations in time. It does not necessarily exclude the possibility to create more or less temporary assumptions of ad hoc generalizations about global issues. It might be assumed as possible to reach systemic knowledge about diverse phenomena.

The strategy for IS proposed here is to be seen as a part of an effort to create an organizational sense-making bridge and oscillating relationship between analysis of a past and design of a future (e.g., Weick 1995).

Rationalization and resistance leads to a possibility not only for crisis but also for a richer communication and change. It is precisely through discussions about communicative action and system that Habermas (1984) tries to create a bridge between the intra-individual system and the interindividual system without giving up the idea of the active and rational individual. The social, cultural, and technical world is populated, created, and recreated by people who in different ways and aspects deal with their relation to the abstract inter- and intra-individual systems in their experienced environment (for related discussions, see Bateson 1972; Berger and Luckman, 1966; Latour 1999).

It is (in this paper) assumed, as Giddens (1984, 1991) suggests, possible and meaningful to create and design a future life space with the purpose and efforts to solve different social and cultural problems. It should also be seen as meaningful to create a gender strategy as an integral part of an IS analysis strategy. The main dimension focused on in this paper has to do with uniqueness ignorant theories and practices. A naive understanding of IS, organizational theories, and practices might inhibit a more adequate and progressive understanding of an unconsciously regenerative blinding behavior. 


\section{CONCLUSION}

The IS discussion needs to take into consideration and support a greater awareness of contextual dependencies and their potential organizational and social implications. When engaging in efforts to help individuals to combine their (work and family) roles to advance organizational change and benefits, enquiries into contextual dependencies become increasingly important. Also, given that IS research practice has often treated even basic individual and organizational issues as being either nonexistent or nonrelevant in the IS arena, there is a need to expand our field to include a contextually relevant strategy.

The whole point with this discussion on contextual dependencies is to intervene in a (biased) IS community and to support an active reevaluation and reconsideration of our practical and theoretical environment. IS research can be seen as in serious need to avoid being weak on the self-critical motivational issues, and the issues of ethics and culture. An enhanced version of contextual analysis might be seen as a possible way forward.

\section{REFERENCES}

Argyris C. Overcoming Organizational Defenses: Facilitating Organizational Learning, Englewood Cliffs, NJ: PrenticeHall, 1990.

Argyris, C., and Schön, D. A. Organizational Learning, Reading, MA: Addison Wesley, 1978. Argyris, C., and Schön, D. A. Organizational Learning II Theory, Method and Practice, Reading, MA: Addison Wesley, 1996.

Bateson G. Steps to an Ecology of Mind, New York: Ballantine, 1972.

Bednar P. M. "A Contextual Integration of Individual and Organizational Learning Perspectives as Part of IS Analysis," Informing Science Journal (3:3), 2000, pp. 145-156.

Berger, P. L., and Luckmann, T. The Social Construction of Reality. A Treatise in the Sociology of Knowledge, New York: Doubleday, 1966.

Galbraith, J. R.; Lawler, E. E.; and Associates. Organizing for the Future: The New Logic for Managing Complex Organizations, San Francisco: Jossey Bass Publishers, 1993.

Giddens, A. The Constitution of Society, Cambridge, MA: Polity Press, 1984.

Giddens, A. Modernity and Self Identity: Self and Society in the Late Modern Age, Stanford, CA: Stanford University Press, 1991.

Habermas, J. The Theory of Communicative Action, Boston: Beacon Press, 1984.

Latour, B. Pandora's Hope: Essays on the Reality of Science Studies, Cambridge, MA: Harvard University Press, 1999.

Walsham, G. Interpreting Information Systems in Organizations, Chichester, England: Wiley, 1993.

Walsham, G., and Sahay, S. "GIS for District Level Administration in India: Problems and Opportunities," MIS Quarterly (23:1), 1999, pp. 39-66.

Wajcman, J. "Feminism Facing Industrial Relations in Britain," British Journal of Industrial Relations (38:2), 2000, pp.183-201.

Weick, K. Sense Making in Organizations, Thousand Oaks, CA: Sage Publications, 1995. 


\begin{abstract}
ABOUT THE AUTHOR
Peter M. Bednar is a senior lecturer at Portsmouth University, UK, and affiliated with the department of Informatics at Lund University, Sweden. His research interest covers areas such as contextual analysis, systems thinking, and information systems development. Apart from working at teaching and research institutions, he has several years of industrial experience. He can be reached at peter.bednar@ics.lu.se.
\end{abstract}

